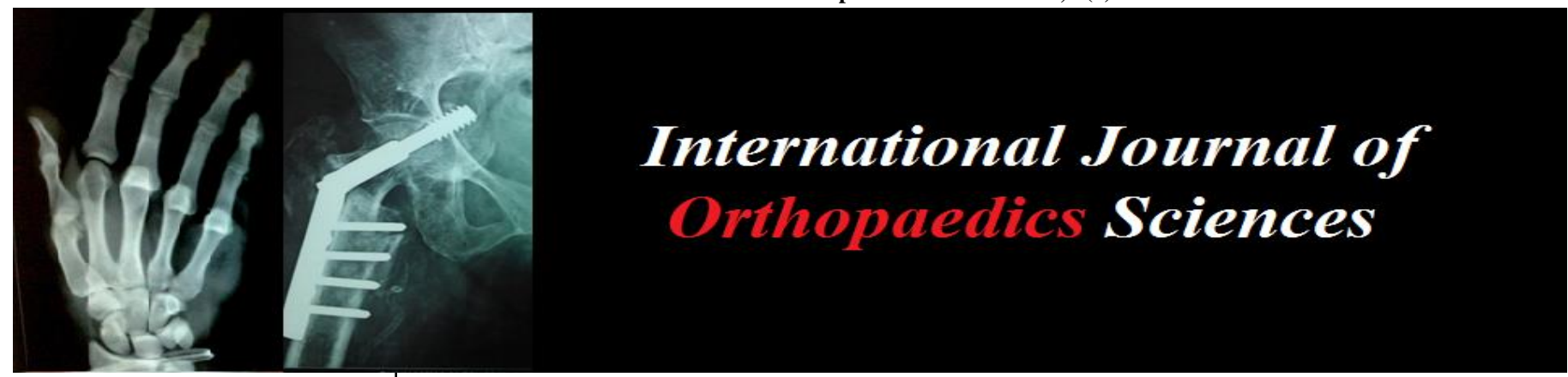

E-ISSN: 2395-1958

P-ISSN: 2706-6630

IJOS 2019; 5(4): 188-191

(C) 2019 IJOS

www.orthopaper.com

Received: 14-08-2019

Accepted: 18-09-2019

Dr. Aluka Sundeep Kund Reddy Assistant Professor, Department of Orthopaedics, Nizam's Institute of Medical Sciences, Hyderabad, Telangana, India

Dr. Bodla Arvind Kumar Assistant Professor, Department of Orthopaedics, Nizam's

Institute of Medical Sciences, Hyderabad, Telangana, India

Dr. P Chandrasekhar Professor, Department of Orthopaedics, Nizam's Institute of Medical Sciences, Hyderabad, Telangana, India
Corresponding Author: Dr. Bodla Arvind Kumar Assistant Professor, Department of Orthopaedics, Nizam's Institute of Medical Sciences, Hyderabad, Telangana, India

\section{Radiographic joint line evaluation and posterior femoral condylar offset using lateral view radiographs of the knee}

\author{
Dr. Aluka Sundeep Kund Reddy, Dr. Bodla Arvind Kumar and Dr. P \\ Chandrasekhar
}

DOI: https://doi.org/10.22271/ortho.2019.v5.i4d.1671

\begin{abstract}
Background: The purpose of this study is to formulate a simple and effective method of calculating native joint line and Posterior Condylar Offset (PCO) in proper lateral modern lateral radiographs of knee joint with minimal magnification coefficient.

Methods: Knee joint line is measured from a. Posterior condylar flare and b. Tibial tuberosity. Line (A) was drawn from posterior cortex of femur that crossed the flare of posterior condyles. Joint line was measured by a perpendicular line (B) from the proximal most point of tibial tuberosity to the line joining the posterior cortex femoral line on lateral view. Posterior condylar offset: PCO was measured (B) relative to the tangent of the posterior cortex of the femur. The ratio was calculated from these measurements in relation to KSS also. Patients attending to Nizam's Institute of Medical Sciences, Hyderabad, in orthopaedic OPD with ideal body mass index who were evaluated for knee radiographs are included in the study after taking proper consent $(n=210)$. Insta RIS PACS software is used to measure the parameters.

Results: The mean joint line measured was $16 \mathrm{~mm}$ (range 14 to 18 ) with 3.0 as standard deviation and the mean PCO was $25 \mathrm{~mm}$ (Range 19 to 31) with a standard deviation of 3.0. The KSS score had a mean of 85 (Range 80 to 90) indicating excellent score with standard deviation of 2.0. Joint line ratio was $95 \%$ Confidence interval (CI) and the ratio for PCO was also 95\% CI. There was a significant difference in the measured joint line between genders.

Conclusion: Using the current measuring method related to standard fixed bony land marks (Posterior femoral line, posterior femoral condyle and tibial tuberosity) which are unaffected even post-surgery the restoration of native joint line and PCO is reliable and reproducible.
\end{abstract}

Keywords: Knee joint line, PCO, KSS, TKR

\section{Introduction}

TKR (Total Knee Replacement) has become one of the prime surgeries of Orthopaedics in India, which has rapidly increased in last two decades with varied satisfaction range. With the advent of newer surgery techniques, modern instrumentation and clear concepts of surgical calculations, preservation of original joint line is coming into reality. As the number of primary TKR are increasing, the rate of revision TKR are also in sudden raise last decade and will continue to increase in near future. It is also a well-known fact that functional outcome of revision TKR is inferior when compared to primary TKR surgery.

The functional outcome has been directly attributed to the Joint line position in a primary TKR [1]. Poor functional outcome have been attributed to revision TKR's in which joint line has not been restored ${ }^{[2]}$. With the elevation of joint line there is increase in patellofemoral joint contact forces with altered quadriceps function and also has change in centre of rotation of knee joint, which is a well-documented fact in the literature ${ }^{[3]}$.

PCO (Posterior femoral Condylar Offset) restoration is a key step of TKR, which provides flexion stability as well as range of movement ${ }^{[5-6]}$. In revision TKR it is difficult to restore the PCO due to posterior femoral condyle bone loss, thus resulting in a small femoral component [7]. We will have to use a thicker polyethylene insert in order to balance both the flexion and extension knee gaps, which will cause elevation of the knee joint line ${ }^{[6]}$. 
In ability to restore the PCO will also affect the outcome of revision TKR.

In recent times Johal et al. ${ }^{[14]}$, Nicolas et al. ${ }^{[15]}$, proposed a simple method of measuring the PCO and native joint line prediction on a lateral radiograph of the knee. With this reference we wanted to incorporate some modifications in the technique and to study outcomes of primary TKR and revision TKR which can serve as reference points using simple lateral view radiographs of the knee and to formulate practical simple acceptable recommendations in regards to joint line alterations and PCO in TKR.

\section{Methodology}

Patients attending to Nizam's Institute of Medical Sciences, Hyderabad, in orthopaedic OPD with ideal body mass index who were evaluated for knee radiographs are included in the study after taking proper consent $(n=210)$. Knee Society Score (KSS) was calculated in all patients. Distal femur and proximal tibial fractures, severe degenerative knee and bone tumours are excluded from the study. This cohort included $120(57 \%)$ male and $90(43 \%)$ female patients with a mean age of 52 years (range 25 to 80 years).

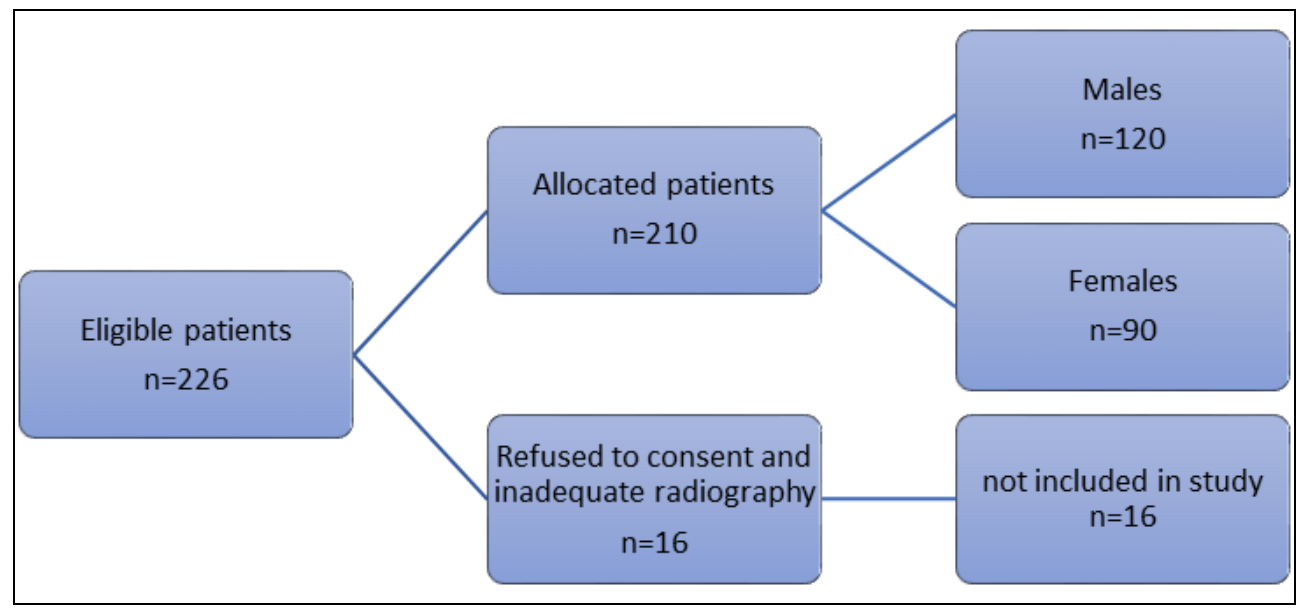

Fig 1: Flowchart of study

\section{Method for taking radiographical lateral view}

Standing [7]: Patient stands parallel to the radiographic cassette, with the lateral aspect touching it and the foot of that knee parallel to the cassette with knee in 30 degrees of flexion. Toe of the back leg touches the heel of the front leg with the back leg locked in full extension. Central ray projection perpendicular to knee.

Patient Lying On Affected Side ${ }^{[8]}$. Pelvis not rotated. Affected knee forward and extend another limb behind it. Epicondyles perpendicular to the $\mathrm{x}$ ray beam. Knee flexion of 30 degrees. Central ray 5 to 7 degrees cephalad at knee joint 1 inch $(2.5 \mathrm{~cm})$ distal to medial epicondyle. Slight angulation of $\mathrm{CR}$ will prevent joint space from being obscured by magnified image of medial femoral condyle. In lateral recumbent position, medial condyle will be slightly inferior to lateral condyle.

Lateral view radiographs from a distance of about $100 \mathrm{~cm}$ from collimeter to the cassette will be taken with above mentioned protocol. Insta RIS PACS software is used to measure the parameters. A standard radiopaque ball of size $50 \mathrm{~mm}$ is placed on the cassette to calculate magnification. All measurements were made using the graphics tools available on insta RISPACS picture archiving software communication system.

A work sheet was created in which the patient's details, measurements are documented. The measurements of the same radiographs are repeated one week later in a random order and documented. Statistical analysis was done with unpaired student's $t$ test for multiple variabilities and outcome.
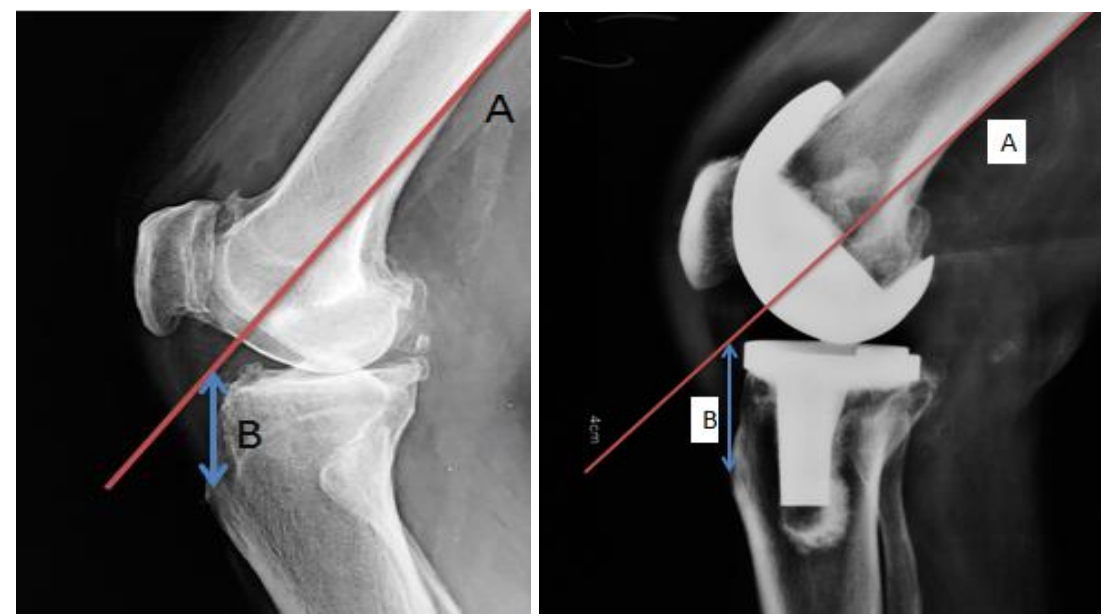

A. Line (A) was drawn from posterior cortex of femur that crossed the flare of posterior condyles.

B. Joint line was measured by a perpendicular line (B) from the proximal most point of tibial tuberosity to the line joining the posterior cortex femoral line on lateral view.

Fig 2: Knee joint line is measured from a. Posterior condylar flare and b. Tibial tuberosity. 

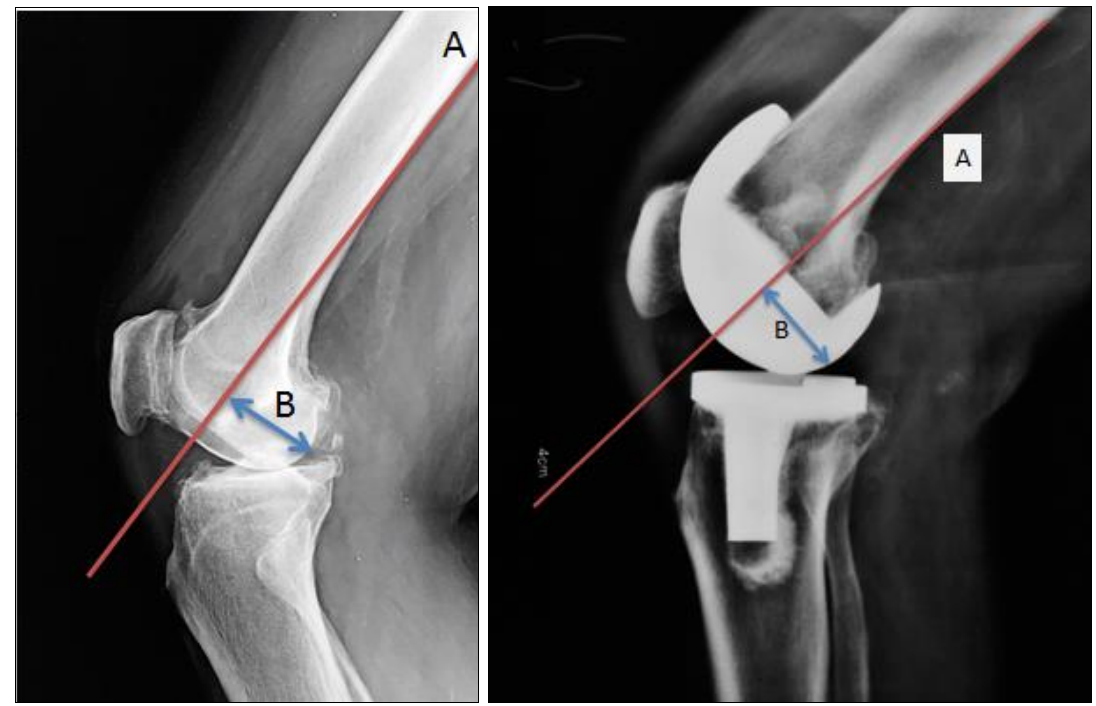

Fig 3: Posterior condylar offset: PCO was measured (B) relative to the tangent of the posterior cortex of the femur.

\section{Results}

Lateral Radiographic measurements were tabulated (Table 1). The mean joint line measured was $16 \mathrm{~mm}$ (Range 14 to 18 ) with 3.0 as standard deviation and the mean PCO was $25 \mathrm{~mm}$ (Range 19 to 31) with a standard deviation of 3.0. The KSS score had a mean of 85 (range 80 to 90 ) indicating excellent score with standard deviation of 2.0 .

Table 1: The mean, standard deviation (SD), and range for all radiographic measurements.

\begin{tabular}{|c|c|c|c|}
\hline $\begin{array}{c}\text { Radiographic } \\
\text { measurement }\end{array}$ & Mean (mm) & $\begin{array}{c}\text { Standard } \\
\text { Deviation }\end{array}$ & Range \\
\hline Joint line & 16 & 3.0 & 14 to 18 \\
\hline PCO & 25 & 3.0 & 19 to 31 \\
\hline KSS score & 85 & 2.0 & 80 to 90 \\
\hline
\end{tabular}

Joint line ratio was $95 \%$ Confidence interval (CI) and the ratio for PCO was also $95 \% \mathrm{CI}$. There was a significant difference in the measured joint line between genders (Table 2). However, there was no significant difference in the ratios for joint line or PCO. In this series the KSS score was not affected by the alteration of joint line and PCO in both genders. KSS scores in this series showed excellent results.

Table 2: Radiographic measurements and ratios for genders.

\begin{tabular}{|c|c|c|c|c|c|}
\hline & $\begin{array}{c}\text { Male } \\
(\mathbf{n = 1 2 0})\end{array}$ & $\begin{array}{c}\text { Female } \\
(\mathbf{n = 9 0})\end{array}$ & Difference & 95\% CI & $\boldsymbol{P}$ value \\
\hline Joint line & $16.8(4.5)$ & $14.7(4.8)$ & 3.6 & 0.1 to 7.1 & $<0.001$ \\
\hline PCO & $28.7(3.1)$ & $22.4(2.8)$ & 1.8 & 0.4 to 0.8 & $<0.001$ \\
\hline KSS score & 86 & 82 & - & - & $<0.001$ \\
\hline
\end{tabular}

\section{Discussion}

The current study showed a modified reliable method of measuring joint line and PCO with the usage of simple lateral radiographs of the knee joint corelating both with the clinical outcomes as measured by KSS. Inability to restore the native joint line and PCO will show negative effects on clinical outcome and the same fact is well documented in literature. There was significant correlation between the posterior femoral line with the perpendicular line drawing from the tibial tuberosity with the joint line and PCO. Using this ratio both PCO and joint line could be predicted with in $3 \mathrm{~mm}$ and $2 \mathrm{~mm}$ respectively. There was no significant interobserver and intraobserver difference with reproducibility of this method up to less than $2 \mathrm{~mm}$ (P value $<0.001)$.
The same can be used to post-operative primary and revision TKR to assess the native joint line and PCO and KSS score outcome can be measures and compared accordingly. There are few limiting factors in this study, such as small cohort study of 210 lateral knee radiographs with wide range of age variability, with slight magnification in radiographs altering the measurements, with Indian ethnicity taken into consideration. The population in this study was mainly of Indian origin and this needs further documentation and study with other populations across the globe. Overcoming the limiting factors in this study with small cohort with variable ages and ethnicity consistent reproducibility of our methodology is near accurate with small interobserver and intraobserver difference, which can be relayed upon in reproducing the joint line and PCO with KSS score correlation. Wang et al. ${ }^{[16]}$ had demonstrated similar study stating variations of joint line and PCO in different ethnic groups showing increased PCO in Chinese population when compared to Western population. The similar cannot be compared in children and adolescent groups who may not require revision TKR.

The current study has similar comparative PCO results to that of Johal et al. ${ }^{[14]}$, which can show overall equivalent results with Western population. Latest study by Wang et al. [16] showed increased PCO in Chinese population when compared to Western population and also minor variations in male and female gender. Bellemans et al. ${ }^{[10]}$ extensively studied about the KSS score and joint line level using a cohort of 200 post primary TKR lateral radiographs and corelated with magnification coefficient and found elevation of joint line to mean of $1.6 \mathrm{~mm}$ but with no difference in gender groups. In all the studies, the variables did not change much according to the population assessed. This study used digital picture lateral radiographs with Insta RIS PACS software to measure the parameters which is becoming more popular in medical science when compared to other study series. This digital radiograph has better clarity and contrast can be adjusted for better visual acumen and thereby reducing the chance of poorquality pictures. The standardisation of this method offers a simple and easy way to calculate the joint line and PCO which can overcome the magnification coefficient.

Reproduction of the original joint line and PCO in primary and revision TKR is important to get excellent KSS score. Porteous et al. ${ }^{[6]}$ and Partington et al. ${ }^{[8]}$ studies state that joint line elevation of greater than $6 \mathrm{~mm}$ has led to decreased 
functional outcome with average KSS score. In this case series, the current method of measuring native joint line and PCO offered less than $4 \mathrm{~mm}$ variability, which is reliable and easy to reproduce without any error in magnification coefficient. This can be incorporated for planning of preoperative calculations of complex degenerated TKR surgeries. Thus, restoring near native joint line and PCO to provide mid flexion stability with excellent KSS score and clinical outcome. The increase in PCO has increased range of movement by with less stability by Malviya et al. ${ }^{[9,10,11]}$. using the current measuring method, which is independent of magnification coefficient, we can reproduce similar excellent results. Thus, this is a multifactorial picture in which many factors will affect the outcome of TKR, of which joint line reproduction with in $4 \mathrm{~mm}$ and $\mathrm{PCO}$ with in $5 \mathrm{~mm}$ play a major role.

\section{Conclusion}

Using the current measuring method related to standard fixed bony land marks (Posterior femoral line, posterior femoral condyle and tibial tuberosity) which are unaffected even postsurgery the restoration of native joint line and PCO is reliable and reproducible. This can also be used as preoperative tool to enable surgeon to plan surgery accordingly in selection of inventory prediction of component sizes. Further research is required in various ethnic groups in large cohort studies to standardise the measurement protocols to improve the functional and radiological outcomes.

\section{Conflict of interests}

The authors have no benefits in any form, did not receive any grant or financial aid relating to this study.

\section{References}

1. Culliford DJ, Maskell J, Beard DJ, Murray DW, Price AJ, Arden NK. Temporal trends in hip and knee replacement in the United Kingdom: 1991 to 2006, Journal of Bone and Joint Surgery B. 2010; 92(1):130135. Arthritis 5.

2. NHS Scotland, Scottish Arthroplasty Annual Report 2010. http://www.arthro.scot.nhs.uk/Reports/Scottish Arthroplasty Project Report 2010.pdf.

3. Friedman RJ, Poss R. Revision total knee arthroplasty in patients with osteoarthritis Rheumatic Disease Clinics of North America. 1988; 14(3):537-544.

4. Babazadeh S, Dowsey MM, Swan JD, Stoney JD, Choong PFM. Joint line position correlates with function after primary total knee replacement: a randomised controlled trial comparing conventional and computerassisted surgery, Journal of Bone and Joint Surgery B. 2011; 93(9):1223-1231.

5. Nicholas D, Clement, David F. Hamilton, and Richard Burnett A Technique of Predicting Radiographic Joint Line and Posterior Femoral Condylar Offset of the Knee, Hindawi Publishing Corporation, Arthritis, 2014, Article ID $121069, \quad 5 \quad$ pages http://dx.doi.org/10.1155/2014/121069

6. Porteous AJ, Hassaballa MA, Newman JH. Does the joint line matter in revision total knee replacement? Journal of Bone and Joint Surgery B. 2008; 90 (7):879-884.

7. König, C, Sharenkov A, Matziolis G et al., Joint line elevation in revision TKA leads to increased patellofemoral contact forces, Journal of Orthopaedic Research. 2010; 28 (1):1-5.

8. Partington PF, Sawhney J, Rorabeck CH, Barrack RL,
Moore J. Joint line restoration after revision total knee arthroplasty, Clinical Orthopaedics and Related Research. 1999; 367:165-171.

9. Hoeffel DP, Rubash HE. Revision total knee arthroplasty: current rationale and techniques for femoral component revision, Clinical Orthopaedics and Related Research, 2000; 380:116-132.

10. Bellemans J, Banks S, Victor J, Vandenneucker $\mathrm{H}$, Moemans A. Fluoroscopic analysis of the kinematics of deep flexion in total knee arthroplasty. Influence of posterior condylaroffset, Journal of Bone and Joint Surgery B. 2002; 84 (1):50-53.

11. Malviya A, Lingard EA, Weir DJ, Deehan DJ. Predicting range of movement after knee replacement: theimportance of posterior condylar offset and tibial slope, Knee Surgery, Sports Traumatology, Arthroscopy. 2009; 17 (5):491-498.

12. Massin P, Gournay A. Optimization of the posterior condylar offset, tibial slope, and condylar roll-back in total knee arthroplasty, Journal of Arthroplasty. 2006; 21(6):889-896.

13. Bellemans J. Restoring the joint line in revision TKA: does it matter? Knee. 2004; 11(1):3-5.

14. Johal P, Hassaballa MA, Eldridge JD, Porteous AJ. The posterior condylar offset ratio, Knee. 2012; 19:843-845.

15. Bartko JJ. The intraclass correlation coefficient as a measure of reliability, Psychological Reports. 1966; 19(1):3-11.

16. Wang W, Tsai TY, Yue B, Kwon YM, Li G. Posterior femoral condylar offsets of a Chinese population, Knee, 2013.

17. Tan SL, Lewis RA. Picture archiving and communication systems: a multicentre survey of users experience and satisfaction, European Journal of Radiology. 2010; 75(3):406-410.

18. Phillips JH, Albregts AE, Kling Jr TF, Cohen MJ, Digital radiography in paediatric orthopaedics: A prospective, controlled, and randomized trial, Journal of Paediatric Orthopaedics. 1994; 14(3):377-380.

19. Hofmann AA, Kurtin SM, Lyons S, Tanner AM, Bolognesi MP. Clinical and radiographic analysis of accurate restoration of the joint line in revision total knee arthroplasty, Journal of Arthroplasty. 2006; 21(8):11541162.

20. Laskin RS. Joint line position restoration during revision total knee replacement, Clinical Orthopaedics and Related Research. 2002; 404:169-171.

21. Mahoneyand OM, Kinsey TL. Modular femoral offset stems facilitate joint line restoration in revision knee arthroplasty, Clinical Orthopaedics and Related Research. 2006; 446:93-98.

22. Brilhaultand JM, Ries MD. Influence of offset stem couplers in femoral revision knee arthroplasty: A radiographic study, Knee. 2012; 19(2):112-115. 